\title{
Norois
}

Environnement, aménagement, société

\section{Soleil, tourisme et cuisine : 150 ans de restauration cannoise}

Sun, tourism and cooking: 150 years of catering in Cannes

\section{Rolande Bonnain-Dulon}

\section{(2) OpenEdition}

1 Journals

Édition électronique

URL : https://journals.openedition.org/norois/3584

DOI : $10.4000 /$ norois.3584

ISSN : 1760-8546

Éditeur

Presses universitaires de Rennes

\section{Édition imprimée}

Date de publication : 30 juin 2011

Pagination : 11-22

ISBN : 978-2-7535-1479-9

ISSN : 0029-182X

\section{Référence électronique}

Rolande Bonnain-Dulon, « Soleil, tourisme et cuisine : 150 ans de restauration cannoise ", Norois [En ligne], 219 | 2011, mis en ligne le 30 septembre 2013, consulté le 13 janvier 2022. URL : http:// journals.openedition.org/norois/3584; DOI : https://doi.org/10.4000/norois.3584

Ce document a été généré automatiquement le 13 janvier 2022.

(c) Tous droits réservés 


\title{
Soleil, tourisme et cuisine : 150 ans de restauration cannoise
}

\author{
Sun, tourism and cooking: 150 years of catering in Cannes
}

Rolande Bonnain-Dulon

1 Les occasions de recourir à une cuisine faite en dehors du foyer sont multiples et elles obéissent à des modèles géographiques, sociaux et culturels. Chaque groupe qu'il soit géographique ou social a sa propre gastronomie, évoluant toutefois dans un cadre culturel général. Il fréquente par nécessité ou par choix des établissements qui se différencient par l'architecture, la décoration, les services, la qualité, la présentation des mets... et le prix des prestations offertes. Avec l'avènement de nouvelles élites qui élaborent des manières séparées de consommation et de distinction, les goûts évoluent tout comme les façons de les satisfaire. Les formes urbaines spécialisées qui en sont issues (localisation, concentration et aussi architecture) évoluent également : chaque ville se présente ainsi comme un feuilleté que l'on peut déchiffrer. Il nous a semblé utile de reprendre l'analyse des rapports entre tourisme et restauration pour la Côte d'Azur (Bonnain-Dulon et Brochot, 2008) en changeant d'échelle et en prenant en compte l'espace environnant: Cannes a été choisie car la ville est mondialement connue malgré sa taille relativement modeste et son histoire est exemplaire, illustrant les changements dans sa restauration (offre et contenu) en fonction de clientèles bien différentes. Après l'époque des palaces et de la classe oisive qui y prenait ses quartiers d'hiver, après celle de l'entre-deux-guerres où une relative démocratisation s'associe au triomphe de l'automobilisme et au développement du tourisme, la période actuelle se caractérise entre autres par une mondialisation de l'offre et un héliotropisme qui ne se dément pas. Quelles en sont les retombées sur la ville, sa région et la restauration?

\section{À la recherche du soleil d'hiver}

2 L'histoire de la « découverte » de Cannes par le chancelier britannique Lord Brougham est bien connue. En 1834, se rendant en Italie comme nombre de personnes de l'aristocratie des pays du Nord recherchant le soleil pendant l'hiver, il s'en était vu 
refusé l'accès en raison d'une épidémie de choléra en Provence. Résidant alors à l'Auberge du Relais de la Poste aux chevaux (créée en 1795-1798), en contrebas du Suquet, partie élevée de cette petite localité de 3000 personnes environ, le lord-chancelier fut séduit par le site et décida de s'y installer : il fit construire une imposante villa sur la route de Fréjus à Antibes et attira dans la localité ses riches amis. Jusque-là, cette bourgade de marins et de pêcheurs-paysans, petit port d'approvisionnement et débouché médiocre de l'arrière-pays (Kayser, 1954) vivotait et ne pouvait se développer en raison de la concurrence de Grasse et d'Antibes. Au pied du Suquet, le faubourg de Cannes s'étendait fort peu au delà de la porte du Poussiat (à l'emplacement de l'actuel marché Forville). À l'exception de l'auberge Pinchinat citée supra, les relais de diligence n'offraient que des tables d'hôte plutôt médiocres dont les touristes européens à la recherche des bienfaits du climat devaient se contenter pendant leur voyage avant de goûter à la cuisine familiale de leurs hôtes.

Grâce à sa nouvelle jetée obtenue en 1839 par l'entregent de Lord Brougham, Cannes va devenir le marché actif du canton, se développer et sa population sera de 6000 habitants en 1852. Si un tiers de la population inscrite sur la liste électorale dépend de la mer, du pêcheur au douanier et un quart des inscrits de la liste électorale travaille dans le secteur du petit artisanat à l'exception d'une douzaine d'entreprises traitant des produits provenant du monde agricole (parfumeries, distilleries et savonneries), l'autre quart des inscrits vit de l'agriculture ${ }^{1}$. Cependant, en 1860, avant l'arrivée du chemin de fer, il n'existe encore que trois hôtels pouvant loger les voyageurs : Pinchinat près du rivage, l'Hôtel de la Poste et l'Hôtel du Nord sur la route d'Antibes, les hivernants se contentent alors de la cuisine de leurs hôtes ou se restaurent dans leurs villas².

4 Après 1863 , les touristes à la recherche du soleil d'hiver affluent. Ces riches arrivants se font construire de somptueuses habitations et de nouveaux quartiers à l'ouest et à l'est de Cannes se développent ${ }^{3}$. De véritables colonies temporaires s'installent, anglosaxonnes d'abord, puis russes. De nombreux hôtels ouvrent alors ${ }^{4}$.

5 L'exploitation du dénombrement de $1901^{5}$ nous donne une idée précise de la localisation et de la physionomie de l'hôtellerie cannoise du début du siècle (fig. 1). 
Figure 1 : Cannes en 1899/Cannes in 1899

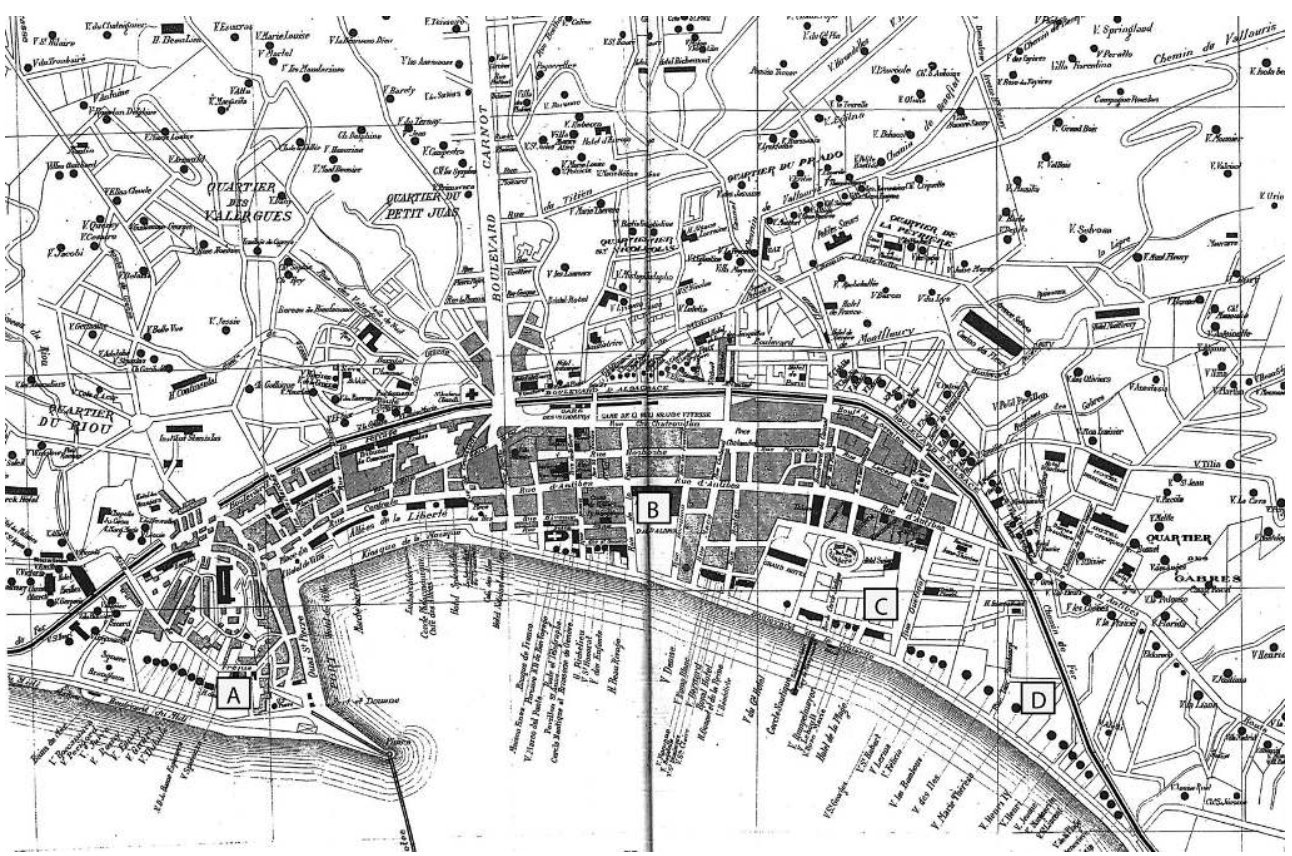

Échelle : 1/55 000 - Légende : A) Emplacement de l'auberge Pinchinat - B) Emplacement du Gray d'albion - C) emplacement du futur Carlton - D) Emplacement du futur Martinez.

6 La ville s'organise le long du rivage où la plage artificielle de la Croisette sera ouverte en 1891. De nombreuses villas y sont édifiées. Quelques hôtels s'installent rue d'Antibes qui deviendra rapidement la rue commerçante de la ville mais les palaces qui feront la renommée de la ville sont érigés dans les collines surplombant l'étroite plaine littorale où les résidents peuvent disposer d'un air pur sans vent, d'une vue sans obstacles sur les îles de Lérins et d'un parc où ils peuvent se promener à loisir et goûter ainsi aux charmes d'une végétation à la fois méditerranéenne et subtropicale. Ces grands hôtels dispensent déjeuners, dîners, et goûters, buffets de réceptions et repas de réveillon à leurs résidents et... à leurs domestiques logés dans les pièces de courrier. Les salles à manger sont à la taille des immeubles qui les abritent ${ }^{6}$. A la même époque, quelques auberges restaurent les voyageurs en transit sur la route de Fréjus à Antibes.

7 Au tournant du siècle, il y a 47 hôtels relevés grâce au registre du Dénombrement: l'hôtellerie cannoise se compose de nombreuses petites structures ${ }^{7}$ et de 14 grands hôtels construits pour 10 d'entre eux avant 1900 (tableau 1$)^{8}$.

Tableau 1 : Classement des hôtels selon le nombre d'employés logés sur place (source : dénombrement de 1901 et de 1931)/Hotels classification according to housed employees' number (1901 and 1931 census)

\begin{tabular}{|l|l|l|l|l|l|l|}
\hline $\begin{array}{l}\text { Nombre d'employés } \\
\text { de l'hôtel }\end{array}$ & $1-5$ & $6-10$ & $11-20$ & $21-30$ & $31-50$ & 51 et + \\
\hline Nombre d'établissements en 1901 & 5 & 12 & 11 & 7 & 7 & 7 \\
\hline Nombre d'établissements en 1931 & 47 & 30 & 12 & 7 & 7 & 3 \\
\hline
\end{tabular}




\section{construit au bord de la mer jugée trop humide et tous sont pourvus d'un parc qui les} prolonge. palaces, ont été construits un peu antérieurement à leurs homologues plus importants qui nécessitaient de réunir des capitaux importants pour leur édification.

Pour gérer ces entreprises, une solide formation professionnelle ou une bonne expérience est requise et la direction est confiée à un professionnel salarié, quelquefois recruté assez loin : c'est le cas 9 fois sur 14. On compte seulement deux Français à la tête de l'hôtel. A clientèle internationale, direction internationale.

11 Pourtant, la réputation de la cuisine de l'Hexagone joue car 9 chefs sur 14 sont français. Quant à la composition de la brigade, logée sur place y compris les pâtissiers suisses, elle confirme la suprématie de la cuisine française : le choix du personnel de l'Hôtel du Prince de Galles est exemplaire : la direction est française, le chef aussi, les pâtissiers suisses, les garçons de cuisine italiens ${ }^{9}$ (tableau 2).

Tableau 2 : Origine des employés travaillant aux cuisines des palaces (source : dénombrement de 1901 et 1931)/Palace kitchens employees'nationality (1901 and 1931 census)

\begin{tabular}{|l|l|l|l|l|l|l|}
\hline Pays & Autriche & Suisse & Allemagne & Italie & France & Autre \\
\hline $\begin{array}{l}\text { Nombre d'employés provenant de ces pays en } \\
1901\end{array}$ & 3 & 13 & 14 & 20 & 32 & 1 \\
\hline $\begin{array}{l}\text { Nombre d'employés provenant de ces pays en } \\
1931\end{array}$ & - & - & - & 8 & 57 & - \\
\hline
\end{tabular}

12 La présence importante de l'aristocratie européenne est l'occasion de nombreuses manifestations et de fêtes. Quant à la cuisine, elle se doit d'être conforme à ce que les clients trouvent chez eux : riche, ostentatoire et française ${ }^{10}$ dans son inspiration car les grands chefs de l'époque sont français et s'exportent de New York à Saint-Pétersbourg. On dispose encore de certains menus rédigés pour les repas quotidiens qui comportent de 5 à 6 services où « la nécessité de varier l'aspect des plats - et donc leur appellation imposait de jouer sur la décoration et le luxe des ingrédients» (Bonnain-Dulon et Brochot, 2008). Ils ne rappellent en rien la province où ils sont dégustés. Donnons un seul exemple, celui du simple dîner du 6 mai 1914 de l'Hôtel de l'Univers : «Consommé printanier royal - Saumon de Loire sauce riche - Pommes nouvelles à l'anglaise Mignonettes d'agneau à la Favorite - Asperges sauce mousseline - Poularde truffée Salade cœur de laitue - Bombe Nélusko - Gâteau Médicis - Fruits. Vins : Graves, Corton, Saint-Perray, Mousseux » (Archives communales de Cannes, 2004)

Comme dans d'autres menus de palaces, les plats renvoient peu à la région où ils sont consommés.

14 Quant aux 17 hôtels qui occupent de 1 à 10 employés, ils sont gérés familialement et n'offrent pas tous les services d'un cuisinier à demeure (celui-ci peut loger en ville ou alors, les clients ont recours aux services d'un traiteur) et quand ils en ont un, il est Français dans 7 cas sur 17. Ici, la réputation de la cuisine française n'est pas le facteur décisif dans le choix de ces établissements modestes. 


\section{7 établissements déclarent employer moins de 11 personnes contre 17 trente ans} auparavant. La gamme moyenne n'a pas bougé. En revanche, s'il y a moins de palaces, 11 contre 14 , ils sont de construction récente et emploient beaucoup plus de personnel. Surtout, ils se rapprochent du bord de mer, pour satisfaire à la nouvelle vogue de la plage et du soleil. Le Carlton (plus de 130 employés) sera érigé en 1910-1911 avec des capitaux fournis en partie par la Société des Bas-Alpins qui ont fait fortune au Mexique, le Majestic (73 employés) voit le jour en 1925, le Martinez en 1929. Dans la catégorie supérieure, le Miramar (1929) rejoint ceux qui l'ont précédé sur la Croisette, pieds dans l'eau et vue sur les îles de Lérins. emploient plus de 312 personnes, un seul est géré par son propriétaire résident, les autres par un directeur appointé. Autre changement dû aux séquelles de la guerre de 1914-1918 : six directeurs sont français, trois sont suisses, un seul est italien. Exit les managers allemands et autrichiens. Ce changement dans le recrutement des directeurs 
et aussi dans la clientèle (elle est désormais française à moitié) se voit dans le choix du chef et la composition de la brigade : des chefs français qui vont satisfaire aux goûts nouveaux sont désormais recrutés dans 8 cas sur 10. La réputation d'Auguste Escoffier natif de Villeneuve-Loubet, assurée par César Ritz (qui s'est marié à Cannes), celle de Joseph Donon également natif de Villeneuve-Loubet, y contribuent. Le personnel de cuisine y compris les pâtissiers est français à $88 \%$. Les cafetiers italiens sont toujours prisés mais il n'y a plus de pâtissiers suisses ${ }^{13}$.

21 En comparant la gestion des palaces avec celle des petites structures (de 1 à 10 employés), on voit que ces dernières sont dirigées dans sept cas sur dix par un Français. L'hôtel ou la pension est tenu par le propriétaire du fonds de commerce ou son gérant, aidé le plus souvent par sa famille. L'établissement n'offre pas de service de restauration hormis celui du petit déjeuner. Les vacanciers s'adressent aux nombreux restaurants qui se sont installés ${ }^{14}$. Et changement notoire dans les arguments ayant présidé au choix du voyage, la cuisine devient désormais un critère essentiel comme on le voit au succès du Guide Michelin.

En ce qui concerne les restaurants « deux étoiles » signalés par le Guide Michelin, ils sont localisés pour moitié dans les Alpes-Maritimes et plus particulièrement dans les stations fréquentées naguère par l'aristocratie et la grande bourgeoisie. Au nombre de 8 , aucun d'eux n'appartient à un hôtel, «le palace fonctionnant encore en autarcie et sur des bases culinaires anciennes» (Bonnain-Dulon et Brochot, 2008). Le menu du 20 octobre servi à l'hôtel du Pavillon porte toujours la marque de la cuisine fastueuse pratiquée avant la guerre de 1914-1918 mais elle s'est allégée : «Huîtres Marennes Consommé double aux profiteroles - Bouchées Reine Margot - Selle d'agneau Renaissance - Petits pois à la Française - Bombe pralinée - Friandises - Fruits - Café Vins : Graves, Médoc, Kressman, Moet, White Star, Liqueurs » (Archives communales de Cannes, 2004).

On note des transformations dans l'offre culinaire des grands hôtels : si en 1913, on ne relevait qu'un « rouget grillé Niçoise " parmi les quatre poissons proposés au menu de l'Hôtel de Californie, quatre légumes méditerranéens les accompagnaient ainsi qu'un dessert de mandarines givrées; en 1939, le même hôtel propose toujours le « rouget grillé » mais il est désormais intitulé « Belle Niçoise » et la carte propose 16 légumes ${ }^{15}$. Les restaurants de la Côte d'Azur, et de Cannes en particulier, offrent toujours une cuisine coûteuse et raffinée à leur clientèle fortunée qui délaisse provisoirement ses villas et ses yachts. En revanche, au niveau immédiatement inférieur du classement du Michelin, on ne compte plus que quatre établissements dans les Alpes-Maritimes sur les 51 distingués par les inspecteurs pour l'ensemble de la France: la cuisine locale ne semble pas pour eux, se signaler par son originalité et sa recherche.

Entre 1901 et 1931, la population de Cannes a gagné $50 \%$ mais la ville n'a pas été la seule à profiter du tourisme : entre 1861 et 1936, Mandelieu et Mougins ont triplé leurs effectifs, accroissement non dû au mouvement naturel mais à l'émigration italienne dans sa majorité ${ }^{16}$. L'attraction des communes littorales et sub-littorales joue aussi dans les limites du département: plus d'un tiers des habitants du canton (à l'exception de Cannes-ville) sont des citadins de la zone littorale, un autre tiers est composé des populations des collines niçoises (Bar-sur-Loup, Cagnes, Vence), le dernier tiers est fourni par les Grassois. La Roquette-sur-Siagne et Pégomas affichent une croissance nette de leurs effectifs, due à une production agricole assurée par une main d'œuvre importée d'Italie. 

âgée $^{18}$, sédentaire pour les $2 / 3$ et aisée. Ses habitants sont propriétaires de leur appartement dans la même proportion que celle constatée pour l'ensemble de la France ${ }^{19}$ mais les appartements ne sont pas tous des résidences principales ${ }^{20}$. Quant au tourisme, on sait que la Côte d'Azur attire annuellement dix millions de personnes dont la moitié sont des Français ${ }^{21}$. En été, Cannes double donc sa population et pendant le reste de l'année, «l'activité congrès " s'étale sur 19 semaines. Les chiffres relatifs à l'hôtellerie en témoignent : les établissements recevant des touristes sont au nombre de 100 en 2009 et totalisent 5282 chambres dont la moitié relèvent d'établissements de 4 étoiles et plus. Ce sont ces derniers qui font la réputation et la renommée de la ville.

Comment Cannes répond-elle aux considérables besoins de restauration hors du domicile de ces deux types de clientèle (la sédentaire et la nomade) et auxquels précisément ? Il n'est que de se promener entre le boulevard Jean Hibert, le Suquet et le Port Canto pour voir que les restaurants s'y pressent nombreux, parfois côte à côte ${ }^{22}$. On annonce 450 restaurants, toutes catégories confondues, Mandelieu et Mougins compris. L'Office du tourisme cannois en dénombre $316 \mathrm{y}$ compris les snacks et les pizzerias. Compte tenu des restaurants de plage et des kiosques le long du rivage qui débitent cafés, boissons fraîches, panini et autres nourritures rapides, on peut estimer qu'il existe 400 établissements servant à manger et qui se ventilent ainsi selon leur propre déclaration : 5 restaurants " gastronomiques ", 5 brasseries "gastronomiques " (de prix et confort moindres que dans la catégorie précédente), 59 restaurants à spécialité étrangère y compris les pizzerias très appréciées par les touristes peu argentés ou pressés et les jeunes, 13 restaurants brasseries, 32 restaurants de plage et 47 restaurants thématiques : seulement 15 d'entre eux déclarent servir de la cuisine provençale. Les autres établissements ne déclarent pas une orientation précise.

30 Ces établissements relèvent de circuits d'approvisionnement très différents. Les grands hôtels en particulier ont leurs centrales d'achat propres. Bon nombre de restaurateurs

Norois, 219 | 2011 
se fournissent chez Metro, hypermarché de gros établi à Mandelieu pour l'épicerie, le vin, les fromages mais aussi pour le poisson, la viande sous vide et les surgelés ${ }^{23}$. Les chefs complètent leurs approvisionnements près des grossistes et des semi-grossistes locaux implantés autour du marché Forville, réputé depuis l'entre deux guerres d'être «le ventre de Cannes».

\section{Le marché Forville et son rôle local}

$\mathrm{Au}$ pied du Suquet, ce superbe marché entouré de bistrots et de commerces d'alimentation très fréquentés le matin est très animé : on y parle encore provençal, on y rencontre ses amis, les touristes le visitent le week-end sous la houlette de leur guide fourni par les bateaux de croisière. Poissonneries, fleuristes, producteurs et revendeurs de toute sorte s'y alignent sur trois allées. Caractéristique de ce marché en pleine ville, pour 41 revendeurs, on compte 39 producteurs, 20 pêcheurs et 15 horticulteurs. Ils ne sont pas toutefois tous présents le même jour, la météorologie étant primordiale pour les pêcheurs ${ }^{24}$ et la saison pour les producteurs de fruits et de légumes.

Encore vers les années 1960, les maraîchers provenant de la plaine de la Siagne étaient majoritaires mais ce n'est plus le cas. Ces derniers viennent désormais depuis Roquebrune-sur-Argens (Var) jusqu'à La Brigue (Alpes-Maritimes). Entre 1979 et 2000, le nombre des producteurs de La Roquette-sur-Siagne a baissé des $2 / 3$ quand la population globale augmentait de $27 \%$. Les cultures se sont modifiées : en 1979, elles étaient surtout légumières pour une superficie de 49 ha contre 14 ha consacrées aux fleurs, elles n'occupent désormais plus que 25 ha pour 36 ha dévolus aux fleurs et autres plantes ornementales. Mouans-Sartoux comptait 67 exploitations en 1979 se consacrant pour 22 ha aux légumes frais et 2 ha aux fleurs. Vingt ans plus tard, quand la population a augmenté de $12 \%$, il n'y a plus que 16 exploitations qui cultivent 5 ha de légumes et 9 ha de fleurs destinés surtout à la population locale. Mougins, commune qui s'est le plus urbanisé et montre une évolution positive de l'emploi (+ 793 emplois en 10 ans, en partie dus aux services hospitaliers et au voisinage de Sophia Antipolis) l'a fait au détriment de l'agriculture puisque ses 117 exploitations ne sont plus que 22 et la part consacrée aux légumes frais est passée de 50 à 7 ha. Quant à Pégomas, autre localité de la plaine de la Siagne qui fournissait le marché de Cannes, sa population a crû de $25 \%$ en 10 ans et ses 80 exploitations en 1979, consacrées pour 43 ha aux légumes et pour 55 ha aux fleurs, ne sont plus que 20, dévolues pour 26 ha aux légumes. L'urbanisation du littoral et les modifications structurelles de la population active et non active, la réorganisation des circuits d'approvisionnement ainsi que les transformations des pratiques alimentaires (comme celles des manières d'habiter avec le recours au fleurissement poussé) ont eu également leur influence les collines de l'arrière-pays même si elles ont été moins touchées par le phénomène des résidences secondaires. Ainsi, Opio qui envoie toujours ses productions à Forville a certes augmenté sa population de $8 \%$ en 10 ans mais a perdu plus de la moitié de ses exploitations agricoles en 20 ans, en particulier ses oliveraies.

L'activité agricole ne se heurte pas seulement aux difficultés d'écoulement de la marchandise et à la tyrannie des horaires de travail. Les jeunes tentés par l'agriculture ne peuvent pas s'installer s'ils n'héritent pas d'une exploitation conséquente et ils ne peuvent acheter car la pression immobilière est forte. De nouvelles constructions sont édifiées constamment entre La Roquette-sur-Siagne et Pégomas au profit des actifs du 
chef-lieu et des retraités attirés par la Côte d'Azur mais ne pouvant acquérir un logement sur le littoral. La zone d'approvisionnement du marché quotidien souffre directement dans son extension et dans le nombre de ses fournisseurs. Quitte à attirer des producteurs de plus en plus lointains comme c'est actuellement le cas : le vendeur de viande d'agneau et de fromage de chèvre du samedi vient déjà de l'Hérault.

Pourtant, le marché Forville reste animé en dépit de ces difficultés, plutôt d'ailleurs comme une attraction et une institution qu'un organisme bien vivant. C'est l'avis de la dynamique vice-présidente de l'Association des producteurs des marchés cannois : «On périclite. Des producteurs, des vrais, on est de moins en moins. En plus, les gens ont pris l'habitude d'acheter hors saison. " Toutefois, après un temps de réflexion et une remarque de l'enquêtrice sur l'affluence continue à son banc bien approvisionné en légumes non traités et aux prix abordables, elle ajoute : "Quand même on y revient. Doucement mais on $y$ revient." Les campagnes sur la consommation quotidienne des légumes et l'écho médiatique des conséquences des pesticides et de l'irrigation trop importante jouent sur les comportements.

Ce ne sont pourtant pas ces campagnes relatives à la santé qui conduisent certains chefs (et non des moindres) à faire leurs courses à Forville, bien avant huit heures du matin. Jacques Chibois de Grasse, Patrick Rambault de Mandelieu, Christian Morisset d'Antibes, chefs distingués par le Michelin et autres guides gastronomiques, côtoient d'autres restaurateurs cannois plus modestes et le propriétaire d'un traiteur très fréquenté de la rue Meynadier (l'ancienne rue Grande de Cannes). Ce recours à la place marchande dans sa fréquence et son importance n'a cependant plus rien à voir avec la situation d'après la guerre où tous les chefs des grands hôtels et des restaurants venaient se servir à Forville. "Les chefs se réunissaient à la porte du Poussiat, choisissaient et se faisaient livrer! Ils demandaient à ma femme des vieilles recettes provençales. "Si les panonceaux indiquant l'origine fermière et locale des produits attirent les particuliers, ils ne sont pas des signes incitatifs pour les chefs. Pour eux, aller à Forville le matin, où les producteurs sont connus et où les produits ont beaucoup moins voyagé est d'abord une signature d'excellence. C'est l'image que l'on donne de son activité qui compte plus que la réalité des pratiques...

\section{Des clientèles diverses et une offre variée de restaurants}

On a vu plus haut que l'offre cannoise en établissements de restauration était importante et variée. Elle correspond exactement aux différents groupes qui viennent dans la ville et qui y vivent : actifs et retraités qui y ont leur appartement; estivants de juillet au début septembre qui ne fréquentent pas régulièrement les restaurants car ils réoccupent leurs résidences secondaires ou leurs résidences de vacances louées à la semaine et déjeunent souvent d'un sandwich à la plage ; assistants aux congrès qui se déroulent toute l'année ; touristes qui visitent la Côte d'Azur.

En fait, il existe trois populations qui vont au restaurant à Cannes mais dont les obligations, les motivations et les attitudes vis-à-vis de la cuisine diffèrent. Dans la première catégorie se trouvent les touristes, du plus simple au plus fortuné et qui sont essentiels pour le maintien des restaurants de plage. Le cadre, le décor, la composition de la carte et les prix sont des facteurs de choix Dans la seconde catégorie, on compte les professionnels qui assistent aux congrès et sont en quête de distraction plutôt que d'expérience gastronomique car ils travaillent en même temps: ils recherchent des 
repas servis rapidement, simples, légers et modernes avec une orientation internationale. Ils représenteraient $60 \%$ de la clientèle qui fréquente les établissements $\mathrm{du}$ Quai Saint-Pierre au rond-point Dubois-d'Angers en passant par la rue Félix-Faure. La dernière catégorie est composite et beaucoup plus locale: elle choisit son établissement dans la gamme elle aussi très variée qui va de la pizzeria au restaurant, $\mathrm{du}$ plus simple au plus luxueux. Or, si la ville a conservé ses grands hôtels aux équipements dernier cri et aux décors somptueux, pour le Guide Michelin, elle n'a qu'un seul restaurant doublement étoilé et seulement 17 établissements qui méritent une notice. Le guide régional Gantié, plus sensible aux plats et aux vins locaux que le guide rouge, donne 30 références et reprend le jugement du Michelin quant à l'excellence. D'après les gestionnaires des grands hôtels, mettre sur pied un tel établissement est une aventure coûteuse pour ses directeurs et hasardeuse. Seul le Martinez avec son restaurant La Palme d'Or a obtenu et conservé cette distinction. C'est finalement assez peu pour la clientèle aisée qui fréquente la baie de Cannes mais on doit tenir compte des deux prolongements du Cannes gastronomique : Mandelieu-La Napoule et Mougins. La première localité citée à six reprises dans le Michelin, compte un "deux étoiles ». Mougins, pratiquement de même importance, jouit en 2008, selon le Michelin, d'un restaurant doublement étoilé et d'un autre à une étoile parmi les cinq établissements cités. Le Gantié reprend globalement ces jugements en citant seulement deux établissements pour Mandelieu et 10 restaurants pour Mougins qui voit ainsi confirmer son orientation gastronomique, la distinguant tout en la rattachant à Cannes et ses paillettes. Le bilan est finalement modeste pour une agglomération de 100000 habitants. Se trouve vérifiée l'assertion désenchantée d'un responsable du personnel d'un hôtel de luxe cannois : "Les gens qui viennent ici ne sont pas des gastronomes, plutôt des fêtards. C'est l'ambiance qui compte plutôt que l'assiette. " La haute cuisine serait-elle chose sérieuse et non compatible avec l'atmosphère de vacances perpétuelles qui règne sur la Croisette?

Pourtant, c'est à la clientèle de cette dernière catégorie que s'adressent les restaurants offrant une cuisine plus «terroir » qu'elle soit provençale ou italienne ou plus exotique et dont la renommée très locale est assurée par le bouche-à-oreille pour les plus âgés et par Internet pour les plus jeunes. Nous ne parlons pas ici des établissements qui servent une cuisine stéréotypée dont les plats se retrouvent de Marseille à Menton mais des restaurants qui offrent une façon originale de cuisiner, une variété dans la carte et le respect des saisons. Ce ne sont pas toujours des établissements luxueux et de grande taille mais ils satisfont une clientèle fidèle qui va très souvent au restaurant par lassitude de cuisiner et pour y rencontrer les amis. C'est pour elle qu'un jeune chef, ayant fait ses classes à Monaco, a ouvert un restaurant de 33 couverts près du marché et qui a rencontré le succès en deux ans. «Ma clientèle, ce sont des jeunes mais surtout des retraités qui prennent le temps de bien manger, qui savent reconnaître la cuisine maison et les produits frais plutôt que le décor. Les très jeunes gens préfèrent Le Carré d'or (les rues derrière la Croisette) avec les bars à néon. " Il a fait preuve d'une politique commerciale prudente avec un menu d'appel à moins de $20 €$. À sa carte, on trouve les grands classiques de la cuisine provençale (fleurs de courgettes farcies, légumes locaux, préparations à base de farines de légumineuses) et des recettes amusantes et à la mode comme la salade de fruits avec un granité de verveine ou le tiramisu au Nutella. "J'aime travailler les produits du pays de façon assez simple pour les mettre en valeur. Je ne cherche pas à faire 10000 saveurs sur le même produit ». Il a ouvert également un atelier de cuisine à partir de ses recettes. 
39 En fait, c'est l'emplacement qui désigne le type de cuisine devant satisfaire les besoins d'une catégorie précise de la clientèle. Les Cannois permanents ou de passage savent que les petits restaurants plus créatifs ou couleur locale se retrouvent en partie au Suquet, le long de la rue Saint-Antoine ${ }^{25}$. Les restaurants classiques ou à spécialité de poisson et crustacés se regroupent du boulevard Jean Hibert au quai Saint-Pierre et rue Félix-Faure. Les brasseries s'égrènent des Allées de la Liberté vers la Croisette sans déborder du front de mer. Les établissements plus onéreux se rencontrent du Palais des Congrès au Port Canto. On retrouve là des constantes dans la localisation des établissements : au quartier ancien, les restaurants qui se réclament d'une tradition vraie ou simplement affichée, aux quartiers plus récents, la restauration plus classique et aussi plus rapide. Toutefois, la cuisine souffre de la comparaison avec son hôtellerie de renommée mondiale. Différant en cela des Niçois qui expriment - entre autre - leur revendication identitaire par un fort attachement à leur cuisine régionale, la référence des Cannois à la Provence est vague et s'efface derrière celle donnée par la Côte d'Azur, plus récente, plus parlante, plus attractive, s'exprimant davantage par le choix de certains produits consommés dans un site exceptionnel.

\section{Voir et être vu}

40 Autre facteur que la carte pour le choix d'un restaurant, l'existence d'une terrasse prolongeant la salle de restaurant et la remplaçant souvent. Ce n'est pas l'annexe bien connue du café avec ses tables volantes et ses chaises cannées, où l'on se pose à tout moment de la journée, où l'on se donne rendez-vous, où l'on bavarde, celle qui fait le charme des établissements français pour les touristes étrangers. Celle-ci est provisoire et autorisée. À Cannes, la terrasse est très souvent une installation permanente, un abri empiétant sur le trottoir, sur une estrade, avec des murs et un toit en matière plastique souple, qui peut être chauffée à l'occasion. Et bien souvent, l'arrière-salle est vide alors que la terrasse est bondée. Elle signe la station balnéaire. "Quand on vient à Cannes, c'est pour manger dehors. " Tous les restaurateurs en ont une ou fantasment sur ses avantages. On était habitué à ces empiètements qui offraient disait-on l'avantage de moduler l'offre en cas d'affluence quand en 2009, la mairie a voulu faire observer le nouveau règlement réservant $1,40 \mathrm{~m}$ de trottoir aux piétons. Après une crise grave suivie d'âpres négociations, syndicats et autorités sont arrivées à un accord en autorisant un délai aux restaurateurs pour se mettre en règle.

41 Certes, la terrasse autorise à prendre ses repas en plein air avec en prime la magnifique vue de la mer, du port et des bateaux, ce n'est pas là sa fonction essentielle : elle est avant tout un poste d'observation qui permet de voir les passants et d'être vu, surtout en périodes de Festival et de vacances.

Cette utilisation de la terrasse, l'attachement des clients à ce prolongement montre que si Cannes est peu provençale, elle relève bien de cette culture du Sud dont le genre de vie et les habitudes se différencient nettement (Fabiani, 2006), par l'effacement progressif des frontières entre le dedans et le dehors de la maison, l'attrait pour les manifestations du corps, la sociabilité ludique et festive. Outre le goût pour les terrasses, on retrouve tous ces traits dans la vie cannoise : la fréquentation assidue de la rue d'Antibes le samedi, les apéritifs « dînatoires» sur les balcons ou au bord des piscines des villas des alentours, les réseaux amicaux structurés en "bande » qui 
réactualisent leurs liens régulièrement. C'est sans doute pour ces raisons que les Italiens s'y plaisent tant.

Depuis son invention sous la Monarchie de Juillet et comme pour nombre de stations balnéaires, thermales ou sportives du xIx et de la moitié du xxe siècle, Cannes a attiré beaucoup de monde venant d'horizons sociaux, culturels et géographiques divers qu'il a fallu loger d'abord, restaurer ensuite. Son offre gastronomique (nombre d'établissements et type de cuisine) s'est adaptée à ces clientèles. Elle a donc varié selon les époques et les goûts. Avant la guerre de 1914-1918, les « hivernants » résident dans des palaces où la cuisine est surtout d'inspiration française et dans leurs villas avec leurs propres cuisinières. Entre les deux guerres, le tourisme qui profite du soleil d'été et de la plage se démocratise relativement. Les hôtels se multiplient ainsi que les restaurants et la cuisine devient un critère essentiel dans le choix du voyage. Elle se transforme en s'allégeant et en reflétant davantage l'éventail des productions locales. Elle garde toutefois pour un certain nombre d'établissements son côté fastueux hérité de la période d'avant guerre. Les communes agricoles de l'arrière-pays continuent à se développer grâce au marché cannois... Ce qui n'est plus le cas aujourd'hui car l'urbanisation, grande dévoreuse d'espaces à construire, les empêche de maintenir ce rôle. Pour assurer l'activité de ces hôtels et de ces restaurants en dehors de la saison d'été, on mise sur le tourisme d'affaires et la restauration qui lui convient (rapidité, simplicité) ${ }^{26}$. La coloration azuréenne (des plats et des modes de cuisson) est surtout mise en avant dans les restaurants gastronomiques pour satisfaire à la mode du terroir (alors que les produits viennent de plus en plus loin) et elle est plus souvent incantatoire que réelle pour les autres établissements. L'offre locale de restaurants «typiques » regarde désormais vers d'autres parties de la Méditerranée.

\section{BIBLIOGRAPHIE}

ARChives Communales DE CANNES, 2004, Chronique de l'hôtellerie cannoise, Publications des Archives communales de Cannes, $240 \mathrm{p}$.

BonNAIN-Dulon R., BROCHOT A., 2008. Haut-lieu touristique, haute gastronomie et haute clientèle : le tiercé gagnant de la Côte d'Azur?, dans CSERGO J. et LEMASSON J.-P. (dir.), Voyages en gastronomies. L'invention des capitales et des régions gourmandes, Paris, Autrement (Mutations, 250), p. 90-101. BORGIELLA-FABRE O., 1958. L'évolution du tourisme à Cannes depuis la $1^{\text {re }}$ Guerre mondiale, Bulletin de Géographie d'Aix-Marseille, tome LXIX, nº 4, p. 53-83.

BOYER M., 2002. L'invention de la Côte d'Azur, La Tour-d'Aigues, L'Aube, 379 p.

CSERGO J., LEMASSON J.-P. (dir.), 2008. Voyages en gastronomies. L'invention des capitales et des régions gourmandes, Paris, Autrement (Mutations, 250), $263 \mathrm{p}$.

FABIANI J.-L., 2006, Beautés du Sud. La Provence à l'épreuve des jugements du goût, Paris, L'Harmattan, $146 \mathrm{p}$. 
GANTIE J., 2009, Le Guide Gantié, Provence-Côte d'Azur, Ligurie et Piémont-Les tables du Sud, Nice, ROM, $540 \mathrm{p}$.

KNAFOU R., 2008. « Entre lieux et pratiques : réflexions d'un gastronome amateur » dans CSERGO J. et LEMASSON J.-P. (dir.), Voyages en gastronomies. L'invention des capitales et des régions gourmandes, Paris, Autrement (Mutations, 250), p. 127-135.

KAYSER B., 1954, Cent ans d'expansion cannoise. La campagne devant l'urbanisation, Cannes, Imp. Devaye, $48 \mathrm{p}$.

FONDATION AuguSTE EsCOFFIER, 1998, La cuisine de palace, Actes de la première session du colloque de la fondation Auguste Escoffier, décembre 1997, Villeneuve-Loubet, 119 p.

MOUSTIER Ph., 2007. Terroirs : caractérisation, développement territorial et gouvernance, dans CONSALES J.-N. et MOUSTIER Ph. (dir.), Terroirs : caractérisation, développement territorial et gouvernance, actes du colloque de 2006, publié dans Méditerranée, revue géographique des pays méditerranéens, 109, 2007/2, p. 7-8.

\section{NOTES}

1. Au moment de la confection des cadastres 1825-1830, le canton de Cannes qui compte environ 1500 ha, voit $30 \%$ de sa superficie occupée par les terres labourables, $24 \%$ par les vignes seules, $7 \%$ par les oliveraies et $5 \%$ par les vignes comprenant des oliviers.

2. Un seul hôtel est digne de ce nom selon Prosper Mérimée (Chronique de l'hôtellerie cannoise, 2004).

3. Alors que la population résidante est d'un peu plus de 30000 habitants en 1901, la population des hivernants est de 22500 personnes à la fin du siècle.

4. Le Guide Jacob en compte 61 pendant la saison 1884-1885 (Chronique, op. cit.).

5. Archives communales de Cannes 1F 17.

6. Lorsque l'hôtel Continental sera vendu par appartements après la guerre de 1939-1945, la salle à manger et ses dépendances deviendront à elles seules l'Hôtel du Cheval Blanc, rue Guy-de-Maupassant.

7. Ce sont souvent des villas construites pour de riches particuliers entre 1870 et 1880 comme celles du boulevard d'Alsace.

8. À l'époque, 700 à 800 villas sont en location et le tiers des villas cannoises sont propriété des hivernants. Toutes ont leur propre personnel dont bien souvent une cuisinière.

9. Toutefois quand le chef est transalpin, il choisit une brigade de même nationalité.

10. Dans ces palaces, les clients peuvent choisir eux-mêmes leur menu. S'ajoute la préparation à la demande de banquets.

11. En 1928, Cannes recevait autant de touristes étrangers que de Français, en 1938, elle n'accueillera plus que $30 \%$ d'étrangers.

12. ACC, 1 F 23.

13. Certains vont s'installer à leur compte en ville.

14. Ils étaient 45 en 1922 et bientôt presque 200 en 1936 (Chronique..., op. cit., p. 17).

15. Les desserts restent identiques. 
16. Les Italiens représentent $29 \%$ de la population du canton, Cannes exclue à cette date.

17. C'est un phénomène relativement ancien. Déjà en 1906, la population de Cannes était composée à $13 \%$ de personnes provenant du département, en particulier des cantons de Breil et de Sospel.

18. La tranche d'âge la plus importante : près d' $1 / 5^{\mathrm{e}}$ de la population est celle des $60-74$ ans.

19. Les appartements sont des résidences secondaires pour $38 \%$.

20. C'est pendant la période s'étendant de 1950 au premier choc pétrolier que ce parc a augmenté de $50 \%$ et cet élan s'est poursuivi pendant les 25 années suivantes $(+33,2 \%)$, s'accélérant encore de 1990 à 1999 avec le vieillissement de la population.

21. La région parisienne fournit $1 / 3$ du contingent national. La moitié des estivants est composée pour $1 / 5^{\mathrm{e}}$ d'Italiens et $1 / 5^{\mathrm{e}}$ d'Anglais, proportion plus forte que pour l'ensemble de la France qui n'attire que $35 \%$ d'étrangers. Cf. www.touriscope.com.

22. En 2006, le recensement général de la population comptait 155790 actifs pour le bassin d'activité Cannes-Antibes au nombre desquels 11470 travailleurs des hôtels et restaurants soit $7 \%$ du total. $87 \%$ des actifs étaient des salariés. Rappelons qu'en France, les travailleurs des hôtels et restaurants représentent $5 \%$ de la masse des actifs.

23. Les établissements cannois ont depuis l'ouverture du MIN de Nice obtenu une dérogation à l'obligation de s'y fournir.

24. Précisons que ceux-ci réservent le meilleur de leur pêche aux restaurants locaux et aux yachts.

25. En 1950, il n'y avait encore qu'un seul établissement, l'Auberge provençale qui proclame fièrement sa date de création : 1860 .

26. Les réflexions de Rémy Knafou sur les types de localisation des grandes tables (Knafrou, 2008) reliant gastronomie, tourisme et centralité sont particulièrement éclairantes. «La réussite des grandes tables isolées exige le cumul d'un grand nombre de qualités : le talent du chef en premier lieu; une médiatisation réussie...; enfin la capacité du chef à penser le lieu dans sa totalité... Les rares restaurants relevant de cette catégorie sont obligés généralement de fermer en hiver » (Knafou, 2008).

\section{RÉSUMÉS}

Chaque restaurant diffère par sa localisation, son architecture, son service, la nature des mets servis et leur présentation afin de répondre à des clientèles spécifiques. La ville de Cannes en est une bonne illustration car au cours du temps, sa restauration s'est considérablement modifiée en fonction de clientèles bien différentes. Avant la première guerre mondiale, l'aristocratie fréquentait les palaces à la cuisine internationale ; pendant l'entre-deux-guerres, le triomphe de l'automobilisme et le développement du tourisme ont accompagné une relative démocratisation de la fréquentation et une gastronomie plus régionalisée ; enfin, la période actuelle se caractérise par un héliotropisme affirmé et une «méditerranéisation » de l'offre gastronomique. Pourtant, 
loin d'en profiter, la production locale est menacée par une urbanisation galopante. Pour suivre cette évolution, des études de terrain sont associées aux données fournies par les archives.

Each restaurant is different from the others by its location, its architecture, its service appearence and quality of food in order to satisfy specific demands. Cannes has been choosen as an example because the town and its restaurants show clearly the impact of its different customers content since the midth of the 19th century. Before the first World War, aristocraty used to visit palaces with their international cooking; after the first and before the second World War, the triumph of automobilism and the developpement of travel for pleasure went along with a relative democratisation of the frequentation and a new interest for local food and cooking; nowadays, we can observe the search for sunlight and the impact of mediterranean food. However, far to take advantage of these transformations, local agriculture suffers from an uncontrolled urbanization. In order to show this evolution field works are associated with local records

INDEX

Keywords : tourism, gastronomy, local agriculture, catering

Mots-clés : tourisme, gastronomie, production agricole, restauration

\section{AUTEUR}

\section{ROLANDE BONNAIN-DULON}

Centre de recherches historiques (École des Hautes Études en sciences sociales), 54 boulevard Raspail - 75006 Paris, France bonnain@ehess.fr 